\title{
Coadministration of Atorvastatin and Amiodarone Increases the Risk of Pulmonary Fibrosis in Rats
}

\author{
Hamid-Reza Nasria, c Siyavash Joukar ${ }^{b, e}$ Hamid Kheradmand ${ }^{c}$ \\ Hamid-Reza Poursalehi ${ }^{a}$ Shahriar Dabiri ${ }^{d}$ \\ aPhysiology Research Center, Institute of Neuropharmacology, ${ }^{b}$ Cardiovascular Research Center, Institute of Basic \\ and Clinical Physiology Sciences, and Departments of ${ }^{\mathrm{C} C}$ Cardiology, ${ }^{\mathrm{d}}$ Pathology and ${ }^{\mathrm{P}}$ Physiology and Pharmacology, \\ School of Medicine, Kerman University of Medical Sciences, Kerman, Iran
}

\section{Key Words}

Amiodarone $\cdot$ Atorvastatin $\cdot$ Pulmonary fibrosis

\begin{abstract}
Objective: The purpose of this study was to evaluate the effect of atorvastatin administration on amiodarone-induced pulmonary fibrosis in rats. Materials and Methods: Thirty-six male Wistar rats were randomly divided into 4 groups. The control group (CTL) received distilled water $(0.3 \mathrm{ml}$ intratracheally on days 0 and 2 and $0.5 \mathrm{ml}$ orally from day 0 for 3 weeks). The atorvastatin group (AT), in addition to intratracheal distilled water, received $1 \mathrm{mg} / \mathrm{kg}$ of atorvastatin orally from day 0 for 3 weeks. The amiodarone group (AMI) received 2 intratracheal instillations of amiodarone $(6.25 \mathrm{mg} /$ $\mathrm{kg}$ in $0.3 \mathrm{ml}$ of water) on days 0 and 2 and $0.5 \mathrm{ml}$ of distilled water (like the $\mathrm{CTL}$ ). The amiodarone plus atorvastatin group $(\mathrm{AMI}+\mathrm{AT})$ received both these drugs (same doses and methods as for the AMI and AT). After 28 days, the rate of lung fibrosis was estimated according to pathological criteria of lung sections and measurements of hydroxyproline in pieces of left lung tissue. Results: The lung hydroxyproline content was higher in the treated groups (CTL: $0.35 \pm 0.017$, AT: $0.38 \pm 0.012$, AMl: $0.375 \pm 0.018$ and AMI + AT: $0.38 \pm 0.012$ unit/mg protein), but did not reach significance when com-
\end{abstract}

pared with the CTL $(p=0.56)$. Amiodarone administration significantly increased the score of pulmonary fibrosis $(0.5)$ in comparison with the AT $(0.125)$ and CTL $(0)(p<0.5)$. The combination of amiodarone and atorvastatin exacerbated the pulmonary fibrosis $(1.5 ; p<0.01)$ compared to the AMI $(0.5 ; p<0.001)$, AT $(0.125)$ and CTL (0). Conclusion: In this study, the concomitant administration of amiodarone and atorvastatin increased pulmonary fibrosis in rats.

(c) 2015 S. Karger AG, Base

\section{Introduction}

Cardiac arrhythmias are an important cause of mortality in cardiac patients [1]. Several drugs are used for the control and treatment of cardiac arrhythmias. Amiodarone, an antiarrhythmic drug from class 3 , is the most effective and useful agent and is one of the most commonly prescribed drugs in the USA and Europe [2].

Pulmonary toxicity is the major side effect of amiodarone and restricts its use [3]. It has been estimated that about $6 \%$ of people who are taking amiodarone show toxicity associated with its use, which takes the form of intraalveolar or interstitial inflammation or pulmonary fibrosis, and the mortality rate is about $5-10 \%$ [4]. It has been

\begin{tabular}{ll}
\hline KARGER & $\begin{array}{l}\text { () 2015 S. Karger AG, Basel } \\
1011-7571 / 15 / 0252-0150 \$ 39.50 / 0 \quad \text { Karger }\end{array}$ \\
$\begin{array}{l}\text { E-Mail karger@karger.com } \\
\text { www.karger.com/mpp }\end{array}$ & $\begin{array}{l}\text { This is an Open Access article licensed under the terms of the } \\
\text { Creative Commons Attribution-NonCommercial 3.0 Un- } \\
\text { ported license (CC BY-NC) (www.karger.com/OA-license), } \\
\text { applicable to the online version of the article only. Distribu- } \\
\text { tion permitted for non-commercial purposes only. }\end{array}$
\end{tabular}

Siyavash Joukar

Cardiovascular Research Center, Institute of Basic and Clinical Physiology Sciences Kerman University of Medical Sciences

PO Box 7616914115, Kerman (Iran)

E-Mail jokar@kmu.ac.ir, sjokar@gmail.com 
reported that, on average, taking amiodarone for 56 months reduces the total lung capacity and can cause pulmonary fibrosis [5].

Statins are the main drugs for reducing levels of blood cholesterol and are widely used in the treatment of hypercholesterolemia, atherosclerosis and cardiovascular diseases [6]. Soyseth et al. [7] reported that, in addition to the above effects, statins have immunomodulatory properties that can reduce the inflammatory effects of smoking in the lungs of patients with COPD. Bartziokas et al. [8] reported that statins prevent the exacerbation of $\mathrm{COPD}$ and reduce the duration of hospitalization. However, another study demonstrated that statins are not effective for the survival of patients with idiopathic pulmonary fibrosis [9]. A case study showed that one of the side effects of statins could be pulmonary interstitial disease [10]. It is well known that concomitant use of these drugs is inevitable in some patients with cardiovascular disease. To the best of our knowledge, there is no consensus on the beneficial, harmful or null effect of the simultaneous administration of statins on amiodarone-induced pulmonary fibrosis. Therefore, the aim of this study was to investigate the effects of atorvastatin, one of the most commonly used statins in clinical practice [11], along with the intratracheal instillation of amiodarone, on some of the parameters of pulmonary fibrosis in adult male rats.

\section{Materials and Methods}

\section{Chemicals}

Atorvastatin, amiodarone and sodium thiopental were purchased from the Amin Co., Isfahan, Iran, and Sandoz, Kundl, Austria.

\section{Animal Groups}

Experimental procedures were done according to the national guidelines for conducting animal studies (Ethics Committee permission No. KA/92/322, Kerman University of Medical Sciences, Iran) on 36 adult male Wistar rats that weighed $250-350 \mathrm{~g}$, with careful implementation of animal care principles. The animals were randomized into the control group (CTL), amiodarone group (AMI), atorvastatin group (AT) and amiodarone plus atorvastatin group (AMI + AT). The CTL received $0.3 \mathrm{ml}$ of distilled water (vehicle) by intratracheal instillation on days 0 and 2 and $0.5 \mathrm{ml}$ of distilled water orally from day 0 for 3 weeks. The AMI received 2 intratracheal instillations of amiodarone $(6.25 \mathrm{mg} / \mathrm{kg}$ in $0.3 \mathrm{ml}$ of water) on days 0 and 2 [12]. The AT received a low dose of atorvastatin $(1 \mathrm{mg} / \mathrm{kg})$ from day 0 for 3 weeks [13]. The AMI + AT was treated with 2 intratracheal instillations of amiodarone on days 0 and 2 and $1 \mathrm{mg} / \mathrm{kg} /$ day of atorvastatin from day 0 for 3 weeks. The exclusion criterion was if the animals died before the end of 4 weeks. On day 29, the animals were killed following deep anesthe-

Amiodarone plus Atorvastatin Boosts

Lung Fibrosis
Table 1. Histopathological scores showing different degrees of fibrosis in each group

\begin{tabular}{lrlllllll}
\hline \multirow{2}{*}{ roup } & \multirow{2}{*}{$\mathrm{n}^{\mathrm{a}}$} & \multicolumn{7}{c}{ Grade of fibrosis } \\
\cline { 3 - 8 } & & 0 & 1 & 2 & 3 & 4 & 5 & Mean \\
\hline CTL & 8 & 8 & 0 & 0 & 0 & 0 & 0 & 0 \\
AT & 8 & 7 & 1 & 0 & 0 & 0 & 0 & 0.125 \\
AMI & 10 & 5 & 5 & 0 & 0 & 0 & 0 & $0.5^{*}$ \\
AMI + AT & 10 & 0 & 5 & 5 & 0 & 0 & 0 & $1.5^{\ddagger * * *}$ \\
\hline
\end{tabular}

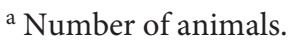

${ }^{\mathrm{b}} 0=$ Normal lung, $1=$ mild fibrous thickening of alveolar or bronchiolar walls, 2 = moderate fibrous thickening of walls without obvious damage to lung architecture, 3 = increased fibrosis with definite damage to the lung structure and formation of fibrous bands or small fibrous masses, $4=$ severe distortion of structure and large fibrous areas ('honeycombing'), 5 = total fibrous obliteration of the field.

${ }^{*} \mathrm{p}<0.05$ vs. the CTL and AT. ${ }^{* *} \mathrm{p}<0.01$ vs. the AMI. ${ }^{\ddagger} \mathrm{p}<$ 0.001 vs. the CTL and AT.

Table 2. The content of lung hydroxyproline in the different groups

\begin{tabular}{lc}
\hline Group $(\mathrm{n}=8-10)$ & Hydroxyproline (unit/mg protein) \\
\hline CTL & $0.35 \pm 0.017$ \\
AT & $0.38 \pm 0.012$ \\
AMI & $0.375 \pm 0.018$ \\
AMI + AT & $0.38 \pm 0.012$ \\
\hline
\end{tabular}

Values are mean \pm SEM. There were no significant differences between groups.

sia induction with thiopental sodium [14]. A piece of lung was removed, washed in saline, frozen in liquid nitrogen and then stored at $-80^{\circ} \mathrm{C}$ for the measurement of hydroxyproline. Thereafter, the rest of the lung was washed with saline infusion and infused with $40 \mathrm{ml}$ of $4 \%$ paraformaldehyde solution. The left lung samples were fixed in $10 \%$ buffered formalin, tissue processing by wash and dehydration steps was done and they were then embedded in paraffin. Lung sections (5- $\mu$ m-thick) were fixed on slides and stained with Masson trichrome. The slides were examined by a pathologist (S.D.) blinded to the animal groups. The severity of lesions was estimated using a previously modified scoring system [15]: $0=$ normal lung, $1=$ mild fibrous thickening of alveolar or bronchiolar walls, 2 = moderate fibrous thickening of walls without obvious damage to lung architecture, 3 = increased fibrosis with definite damage to lung structure and formation of fibrous bands or small fibrous masses, $4=$ severe distortion of structure and large fibrous areas, i.e. 'honeycomb lung', and $5=$ total fibrous obliteration of the field.

Frozen pieces of lung were weighed and then homogenized in $5 \mathrm{ml}$ of $0.1 \mathrm{M}$ Tris-HCl buffer ( $\mathrm{pH}$ 7.4) under ice-cold conditions. 
Fig. 1. Masson trichrome-stained sections of lung tissue in the different groups. a Normal alveoli and bronchioles without specific pathological changes from a CTL animal. b A section from an AT animal showed focal fibrosis in the bronchiolar walls and empty alveolar spaces (white arrows), without obvious fibrosis or macrophages collections in the alveoli. c Increased focal fibrosis in the wall of the bronchioles and also in the alveolar interstitial spaces in an AMI animal (white arrows). The alveolar macrophages are not hyperplastic. d High-power view of increased collagen fibers in interstitial spaces (white arrows) and hyperplastic alveolar macrophages within alveolar spaces (black arrows) in an AMI + AT animal.

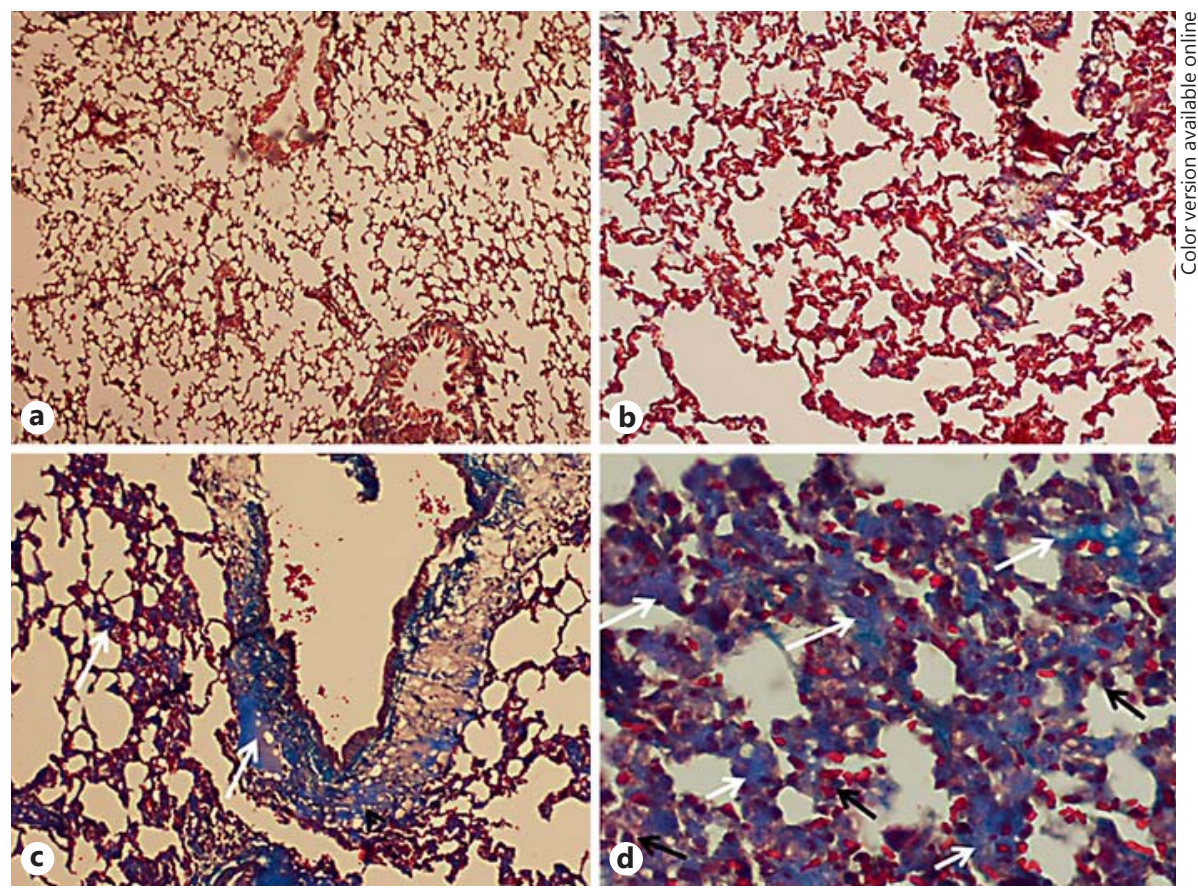

The hydroxyproline value in the lung tissue was measured using the ELISA method and a hydroxyproline kit (Shanghai Crystal Day Biotech Co., Ltd., Shanghai) according the manufacturer's protocol [16].

\section{Statistical Analysis}

The data were reported as means \pm SEM. Comparison of hydroxyproline among different groups was done by 1-way ANOVA and the post hoc Tukey test. Scores of lesions were compared using the nonparametric Kruskal-Wallis test and the Mann-Whitney U test. A p value $<0.05$ was considered statistically significant.

\section{Results}

No rats died during the study period.

\section{Histopathological Findings}

The inspection of the lung tissue revealed normal alveoli without obvious fibrosis or macrophages in the CTL and AT. However, in the AT, mild focal fibrosis in the wall of the bronchioles was seen. Increased focal fibrosis in the wall of the bronchioles and the alveolar interstitial spaces was observed in 5 animals (50\%) in the AMI; this was significant in comparison to the CTL and AT ( $<<0.05$; table 1 ; fig. 1$)$. The lungs of the AMI + AT showed significant changes, i.e. a mild-to-moderate increase in collagen fibers in the interstitial spaces and hy- perplastic alveolar macrophages within the alveolar spaces $(\mathrm{p}<0.01$ vs. the AMI and $\mathrm{p}<0.001$ vs. the CTL and AT; table 1; fig. 1).

\section{Hydroxyproline}

Measurement by ELISA showed higher values of lung tissue hydroxyproline in the AT, AMI and AMI + AT. However, these alterations did not reach a significant level when compared with the CTL (table 2).

\section{Discussion}

In this study, histopathological findings showed that coadministration of atorvastatin and amiodarone exacerbated pulmonary fibrosis in rats, as indicated by the maximum score of fibrosis in the AMI + AT. In addition, intratracheal instillation of amiodarone alone was associated with a mild degree of fibrosis in the lung tissue of some animals. In the clinic, in line with this finding, dose-related pulmonary toxicity is the most important extracardiovascular side effect of amiodarone. Even on a low dose of $\leq 200 \mathrm{mg} /$ day, fatal pulmonary fibrosis may be observed in $1 \%$ of patients [17]. Amiodarone is very extensively used for a wide variety of arrhythmias, especially serious ventricular arrhythmias [17]. Its bioavailability is $35-65 \%$, it undergoes hepatic metabolism 
and desethylamiodarone, as its major metabolite, is bioactive. Its level in the blood is increased by drugs that inhibit the liver cytochrome CYP3A4 enzyme. Higher concentrations of this drug increase both antiarrhythmic effects and the risk of adverse effects [5]. The mechanism of amiodarone-induced pulmonary side effects is not well known. An in vitro study revealed that amiodarone primarily activates necrotic pathways whereas desethylamiodarone activates both necrotic and apoptotic pathways in the culture of human peripheral lung epithelial cells [18]. Other mechanisms that have been suggested include a direct toxic effect and an immune-mediated mechanism [19]. Amiodarone could be associated with macrophage activation and the release of cytotoxic and inflammatory mediators such as TNF- $\alpha$ [20]. These mediators may modulate fibroblast functions such as chemotaxis and the production of collagen, glycosaminoglycans, interleukins, collagenase and prostaglandin $\mathrm{E}_{2}$ [21], and thereby stimulate the process of lung fibrosis.

As mentioned above, this study revealed that coadministration of atorvastatin and amiodarone exacerbates pulmonary fibrosis. Enzyme inhibition is one of the possibilities that could explain the severity of lung fibrosis in the AMI + AT. If 2 drugs sharing metabolism via the same isozyme compete for the same enzyme receptor site, enzyme inhibition may occur and the metabolism of the competing drug would be decreased. This phenomenon can lead to increased serum levels of the unmetabolized drug and consequently its toxicity [21]. With respect to the fact that amiodarone and some statins such as simvastatin, atorvastatin and lovastatin are substrates for liver cytochrome CYP3A4 [22], enzyme inhibition may occur with coadministration of these agents, increasing the chances of toxicity. Consistent with this hypothesis, previous studies have emphasized the adverse events that occur with concomitant amiodarone and statin therapy, especially muscle toxicity. The reason is considered to be the disturbance of the metabolism of cytochrome CYP3A4-dependent statins [23].

Our other finding was the insignificant increase of lung hydroxyproline and mild fibrosis in the tissue in some of the animals, i.e. those that were only treated with atorvastatin. Consistent with this finding, there are several studies that reported that the statins themselves induce the harmful effects in the lung. Lantuejoul et al. [24] reported statin-induced diffuse interstitial pneumonia associated with a nonspecific interstitial pneumonia pattern. A systematic review demonstrated that for every

Amiodarone plus Atorvastatin Boosts

Lung Fibrosis
10,000 reports of a statin-associated adverse event, approximately 1-40 reports were for interstitial lung disease [10]. Walker et al. [25] confirmed that, over a 3-year period, 7 patients who were taking HMG-CoA reductase inhibitors (statins) presented to their respiratory service with interstitial pneumonitis. The mechanism by which statins induce interstitial lung disease is unknown. As thought to be the case in amiodarone-induced lung injury, it is possible that the toxic effect of statins in the lung is mediated by the inhibition of phospholipases which, in turn, produce phospholipid-rich intralysosomal lamellar inclusions in type II lung epithelial cells, histiocytes and endothelial cells, leading to an amphiphilic-drug-like toxic lung injury [24].

Amiodarone and HMG-CoA reductase inhibitors have an amphiphilic structure, an apolar ring and a hydrophilic cationic side chain. It turns out that these structural properties may be related to the development of drug-induced phospholipidosis [26].

Similar to amiodarone, statins have a typical pharmacokinetic pattern, as the tissue/plasma drug ratios rise with the duration of the treatment [27]. This could explain the positive correlation of the pulmonary side effects of these agents and the duration of their use. In addition, as amiodarone induces disturbance of the oxygen consumption in the lung [28], distracting the electron transport chain has been postulated as a potential cause of statin-induced myopathy [29]. Statin-induced lung injury may result from the activation of immune mediators, involving polymyositis [30] and drug-induced lupus, other rare side effects of these agents. As mentioned above, it seems that amiodarone primes macrophages for the production of TNF- $\alpha$ and superoxide. In the presence of statins, these effects may reinforce and lead to an increase in oxidant agents and proinflammatory mediators, thereby triggering the inflammation cascade and causing lung injury.

\section{Conclusion}

Our findings show that the combination of amiodarone and atorvastatin accelerated and enhanced amiodarone-induced lung fibrosis in rats. Further studies are needed to elucidate the involved mechanisms and to confirm these findings in patients. 


\section{References}

$>1$ Lloyd-Jones D, Adams RJ, Brown TM, et al: Heart disease and stroke statistics- 2010 update: a report from the American Heart Association. Circulation 2010;121:e46-e215.

$>2$ Vassallo P, Trohman RG: Prescribing amiodarone: an evidence-based review of clinical indications. JAMA 2007;298:1312-1322.

$\checkmark 3$ Schwaiblmair M, Berghaus T, Haeckel T, et al: Amiodarone-induced pulmonary toxicity: an underrecognized and severe adverse effect? Clin Res Cardiol 2010;99:693-700.

$\checkmark 4$ Reasor M, Kacew S: An evaluation of possible mechanism underlying amiodarone-induced pulmonary toxicity. Proc Soc Exp Biol Med 1996;212:297-304.

5 Kudenchuk PJ, Pierson DJ, Greene HL, et al: Pulmonary effects of amiodarone. J Am Coll Cardiol 1983;1:631.

-6 Young RP, Hopkins R, Eaton TE: Potential benefits of statins on morbidity and mortality in chronic obstructive pulmonary disease: a review of the evidence. Postgrad Med J 2009; 85:414-421.

7 Soyseth V, Breakke PH, Smith P, et al: Statin use is associated with reduced mortality in COPD. Eur Respir J 2007;29:279-283.

$>8$ Bartziokas K, Papaionnou Al, Minas M, et al: Statins and outcome after hospitalization for COPD exacerbation: a prospective study. Pulm Pharmacol Ther 2011;24:625-631.

-9 Nadrous HF, Ryu JH, Douglas WW, et al: Impact of angiotensin-converting enzyme inhibitors and statins on survival in idiopathic pulmonary fibrosis. Chest 2004;126:438-446.

10 Fernandez AB, Karas RH, Alsheikh-Ali AA, et al: Statins and interstitial lung disease: a systematic review of the literature and of food and drug administration adverse event reports. Chest 2008; 134:824-830.

-11 Gu Q, Paulose-Ram R, Burt VL, et al: Prescription cholesterol-lowering medication use in adults aged 40 and over: United States, 2003-2012. NCHS Data Brief 2014;177:1-8.
12 Taylor MD, Van Dyke K, Bowman LL, et al: characterization of amiodarone-induced pulmonary toxicity in F344 rats and identification of surfactant protein $\mathrm{D}$ as a potential biomarker for the development of the toxicity. Toxicol Appl Pharmacol 2000;167:182-190.

13 Chaudagar KK, Mehta AA: Effect of atorvastatin on the angiogenic responsiveness of coronary endothelial cells in normal and streptozotocin (STZ)-induced diabetic rats. Can J Physiol Pharmacol 2014;92:338-349.

14 Joukar S, Zarisfi Z, Sepehri G, et al: Efficacy of Melissa officinalis in suppressing ventricular arrhythmias following ischemia-reperfusion of the heart: a comparison with amiodarone. Med Princ Pract 2014;23:340-345.

15 Ashcroft T, Simpson JM, Timbrell V: Simple method of estimating severity of pulmonary fibrosis on a numerical scale. J Clin Pathol 1988;41:467-470.

16 Binayi F, Joukar S, Najafipour H, et al: The effects of nandrolone decanoate along with prolonged low-intensity exercise on susceptibility to ventricular arrhythmias. Cardiovasc Toxicol 2015, Epub ahead of print.

17 Katzung BG, Masters SB, Trevor AJ: Basic and Clinical Pharmacology, ed 11. New York, McGraw-Hill, 2009, pp 240-241.

18 Black JE: Mechanisms of Amiodarone and Desethylamiodarone Cytotoxicity in Human Lung Cells; Master of Science thesis, 2009 (accessed January 23, 2015). https://qspace.library.queensu.ca/bitstream/1974/5334/1/ black jeanne e 200911_msc.pdf.

19 Papiris SA, Triantafillidou C, Kolilekas L, et al: Amiodarone: review of pulmonary effects and toxicity. Drug Saf 2010;33:539-558.

20 Reinhart PG, Gairola CG: Amiodarone-induced pulmonary toxicity in Fischer rats: release of tumor necrosis factor alpha and transforming growth factor beta by pulmonary alveolar macrophages. J Toxicol Environ Health 1997;52:353-365.
21 Ogu CC, Maxa JL: Drug interactions due to cytochrome P450. Proc (Bayl Univ Med Cent) 2000;13:421-423.

22 Bellosta S, Paoletti R, Corsini A: Safety of statins: focus on clinical pharmacokinetics and drug interactions. Circulation 2004; 109(23 suppl 1):III50-III57.

23 Alsheikh-Ali AA, Karas RH: Adverse events with concomitant amiodarone and statin therapy. Prev Cardiol 2005;8:95-97.

24 Lantuejoul S, Brambilla E, Brambilla C, et al: Statin induced fibrotic nonspecific interstitial pneumonia. Eur Respir J 2002;19:577-580.

25 Walker T, McCaffery J, Steinfort C: Potential link between HMG-CoA reductase inhibitor (statin) use and interstitial lung disease. Med J Aust 2007;186:91-94

26 Lullmann H, Lullmann-Rauch R, Wassermann O: Drug-induced phospholipidoses. II. Tissue distribution of the amphiphilic drug chlorphentermine. CRC Crit Rev Toxicol 1975;4:185-218.

27 Dake MD, Madison JM, Montgomery CK, et al: Electronmicroscopic demonstration of lysosomal inclusion bodies in lung, liver, lymph nodes, and blood leukocytes of patients with amiodarone pulmonary toxicity. Am J Med 1985;78:506-512.

28 Bolt MW, Card JW, Racz WJ, et al: Disruption of mitochondrial function and cellular ATP levels by amiodarone and N-desethylamiodarone in initiation of amiodarone-induced pulmonary cytotoxicity. J Pharmacol Exp Ther 2001;298:1280-1289.

29 Folkers K, Langsjoen P, Willis R, et al: Lovastatin decreases coenzyme Q levels in humans. Proc Natl Acad Sci USA 1990;87:89318934.

30 Liebhaber MI, Wright RS, Gelberg HJ, et al: Polymyalgia, hypersensitivity pneumonitis and other reactions in patients receiving HMG-CoA reductase inhibitors: a report of ten cases. Chest 1999;115:886-889. 